\title{
Multiple AI based Controlling Techniques for Power Filter Output Quality Improvement
}

\author{
Rajesh Raghuvanshi \\ M.Tech Scholar \\ TRUBA College of Science and Technology \\ Bhopal (MP), India \\ raghuwanshirajesh27@gmail.com
}

\author{
Mithlesh Gautam \\ Assistant Professor \\ TRUBA College of Science and Technology \\ Bhopal (MP), India \\ mithlesh.gautam1@gmail.com
}

\begin{abstract}
The excessive of power electronics devices in distribution system has evolved the problem of power quality. This work represents the effective solution of shunt active power filter for eliminating the harmonics to maintain the quality of utility power supply. In the proposed scheme shunt active filter acts as a voltage source and eliminates harmonics by injecting equal but opposite harmonics components at the point of common coupling. Simulation of shunt active filter based on instantaneous reactive has been achieved in the MATLAB/SIMULINK environment. The controllers are being designed with the objective of reducing the THD\% in voltage and current available at the nonlinear load terminal of the system. The controller incorporated optimization algorithms based on the artificial intelligence techniques as well. The comparative analysis of the effectiveness and efficiency of the controllers in improving the quality factors has been carried out. The distortion level in the output voltage waveforms in both the cases being fed to the load when compared, it is found that proposed hybrid structure of control for the filter is more effective in reducing the distortion as compared to standard PQ theory based architecture. The study concluded that the total harmonic distortion in the voltage output waveform being fed to the load using only PQ theory based online learning algorithm is found to be $1.59 \%$ while in case of the output voltage at the load terminal from the power filter using proposed hybrid algorithm comprising of $\mathrm{NN}$ learning with CSA optimizing control and PQ_RLS algorithm for THD reduction the THD\% level is $0.43 \%$.
\end{abstract}

Keywords: Hybrid power filter, CSA, NN, RLS, THD\%.

\section{INTRODUCTION}

Extensive use of non-linear loads leads to a multitude of unwanted miracles in the operation of the electrical system. Of these, the harmonics of current and voltage are the most significant. Generally, an active filter was used to attenuate the harmonics of the mains current. However, they introduce resonance into the system and tend to be bulky. In this sense, active power filters are better known than passive filters because they simultaneously compensate for reactive power and harmonics.

The active power filter topology can be switched in series or shunt or a combination of the two. Active shunt filters are better known than series active filters because the vast majority of sectors require harmonic current compensation. Different types of active filters have been proposed to improve the quality of the electrical system. The characterization depends on the following measurements.

- System parameters to compensate (eg harmonic currents, power factor and harmonic voltages)

- Base current and voltage evaluation technology.

Inverters with a current controlled voltage source can be used with a suitable control system to effectively operate the active power filter.

\section{LITERATURE REVIEW}

V Taraka Rama Reddy et al. [1] Load consumption has increased in recent years, giving electrical engineers a signal on power quality. Due to the large number of non-linear power electronic devices, a utility company is very often prone to harmonic voltage distortions. The purpose of this document is to improve the power quality of the system caused by voltage and harmonic disturbances. Therefore, an integration of the Series Active Power Filter (SAPF) powered by a PV power supply is implemented in this document.

Abdul Kahar et al. [2] the aim of this study is to find the optimal size parameters of the single unlocked coupled filter in the nonsinusoidal system using a new method called Optimization of the distributed ant colony in mixed integers. The optimal value obtained from a single matched filter is used to explain system performance by evaluating other functions. The efficiency of the 
proposed method is demonstrated by comparison with previous publications and other evolutionary computational techniques which are genetic algorithms and particle swarm optimizations.

Arunsankar et al. [3] this paper proposes an efficient hybrid approach using SHAPF to attenuate harmonics in order to maintain PQ in the distribution system. The main objective of the proposed strategy is to improve energy quality by reducing harmonics subjected to the minimum THD. With the proposed approach, EGOA optimizes the data set of fundamental and harmonic loop parameters such as terminal voltage and DC voltage present in the SHAPF based on the variation of the load and the variation of the system parameters.

Buch et al. [4] this paper provides an optimized butterfly flame optimization algorithm to effectively solve Optimal Power Flow problems. The idea of Moth Flame Optimization is driven by the movement of the moth towards the moon. The simulation results of the proposed algorithm are compared with those of other known optimization methods. A comparison of the convergence qualities of the AMFO and the different techniques shows that the AMFO predominates to achieve the optimal current flow solution with fast convergence.

\section{OBJECTIVE}

The prime objective of the project is to reduce distortion level in voltage or current input to the load and at the output of the filter. The research problem is further broken into following subobjectives.

- $\quad$ To design a simple but highly viable hybrid active power buffer that is capable of feeding less distorted voltage to the nonlinear load model.

- $\quad$ To present an optimal controlling of these buffers so as to minimize the voltage distortion by designing a different algorithms for the same that also relay on the artificial intelligence technique.

- Comparing the THD levels of the output voltage waveform with the standard controlling method with the proposed controller design

- Designing of an hybrid controller technique utilizing two optimizing algorithms for the distortion reduction at the voltage line having non-linear load

\section{Design OF SAPF AND ARTIFICIAL INTELligenCE B ASED CONTROL AlgOrithMS}

A. Designing of Active Filter
In this work the power filter design has been obtained using three phase voltage source inverter. For generating this compensating current through active filter, reference currents are generated using reference current generation theories and dc-link voltage of active filter is controlled in order to have less exchange of active power between filter and source.

Following criteria is used for designing filter inductance and capacitance:

1. For making active filter rating low, filter impedance must be as low as possible. Hence, capacitance value must be kept high and inductance value must be kept low.

2. However, a large capacitance value would result into a large amount of capacitive reactive current and a low inductance value of would not suppress the switching ripples caused by the active filter properly.

3. For obtaining attenuation ratio of $10 \%$, the inductance value is kept about 10 times as the supply inductance. Also the passive filter is tuned at a particular harmonic frequency, $\omega r$, the capacitance value is calculated by equation 1

$\omega_{r}=\frac{1}{\sqrt{L_{f} C_{f}}}$

The figure shows the three phase voltage source inverter model that has been designed as a power filter using six MOSFETs having a FET resistance $0.01 \mathrm{ohms}$ and internal diode resistance 0.01 ohms. The hybrid filter used in HPF using conventional 3phase. six pulses have been generated using various algorithms like RLS and modified RLS algorithm which is the combination of both PQ and RLS that has been created in MATLAB/SIMULINK. The pulses drive the gate signals of the MOSFET for a controlled output from them.

\section{B. VSI uses a single LC filter per phase as a passive filter.}

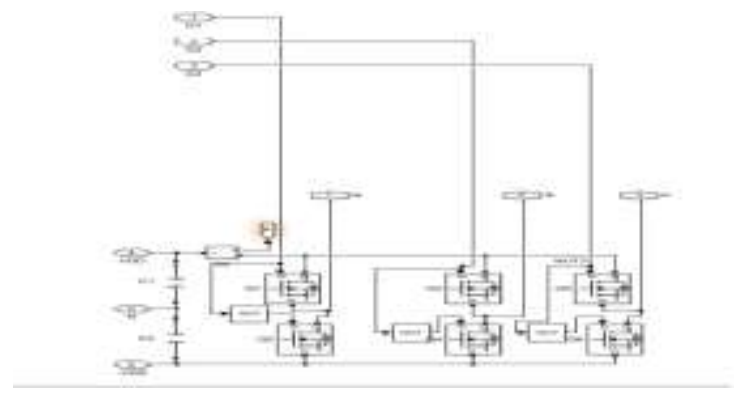

Fig. 1 MATLAB model of Designed inverter

\section{Type of Load}

For stability study, to analyze the stability of a system including a power converter and constant power loads. We need to analyze 
the system stability from the ac side [6], [10]. The analysis was carried out for two different loads. The presented results confirm the effectiveness of the proposed approach, which could be assumed as a very useful tool in the design and analysis of a power generation system. Figure 4.2 shows the loads considered which are non linear load and unbalanced load. This is the load which is considered in all the proposed models for the sake of comparison of efficient and more optimized algorithms.

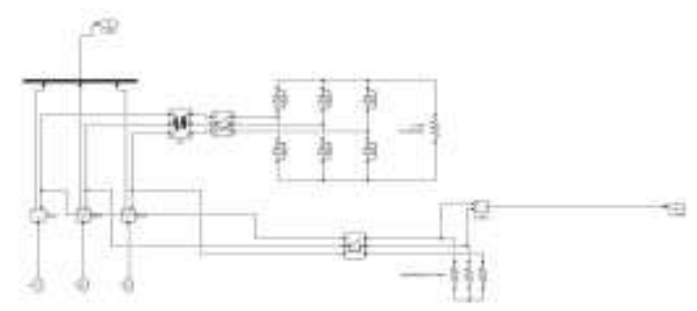

Fig. 2 MATLAB model of 3 Phase Unbalanced Non-Linear Load

\section{Instantaneous Power Theory or PQ-Theory}

In this technique, the three-phase voltage and load currents are transformed into the $\alpha-\beta$ frame and with this the $\mathrm{p}-\mathrm{q}$ frame is obtained. Assuming a three-phase three-wire system, zerosequence component does not exist, so the $\alpha-\beta$ frame can be obtained from the positive and negative-sequence.

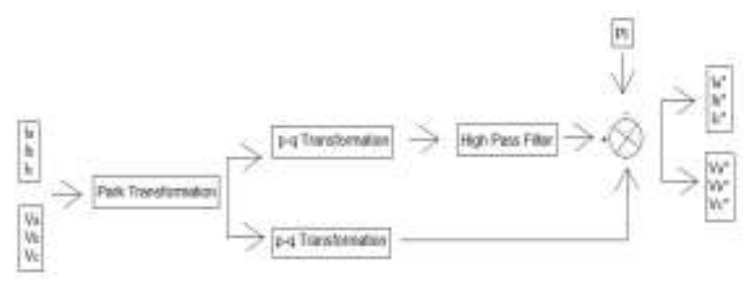

Fig. 3 Flow Chart of PQ algorithm

The instantaneous active and reactive power of the nonlinear load is calculated. The AC component is extracted using high pass filter (HPF) to obtain the compensation reference signals in terms of either currents or voltages. The three-phase reference frame is translated to the orthogonal stationary reference frame.

$\left[\frac{V_{\alpha}}{V_{\beta}}\right]=\sqrt{\frac{2}{3}}\left[\begin{array}{ccc}1 & \frac{-1}{2} & \frac{-1}{2} \\ 0 & \sqrt{\frac{3}{2}} & -\sqrt{\frac{3}{2}}\end{array}\right]\left[\begin{array}{l}V_{a} \\ V_{b} \\ V_{c}\end{array}\right]$
$\left[\frac{I_{\alpha}}{I_{\beta}}\right]=\sqrt{\frac{2}{3}}\left[\begin{array}{lll}1 & \frac{-1}{2} & \frac{-1}{2} \\ 0 & \sqrt{\frac{3}{2}} & -\sqrt{\frac{3}{2}}\end{array}\right]\left[\begin{array}{l}I_{a} \\ I_{b} \\ I_{c}\end{array}\right]$

Instantaneous real power $p$ and reactive power $q$ are given

$\left[\begin{array}{l}p \\ q\end{array}\right]=\sqrt{\frac{2}{3}}\left[\begin{array}{cc}V_{\alpha} & V_{\beta} \\ V_{\beta} & -V_{\alpha}\end{array}\right]\left[\begin{array}{l}I_{\alpha} \\ I_{\beta}\end{array}\right]$
From instantaneous reactive power theory, for obtaining the harmonic component, real and reactive power can be decomposed into $\mathrm{DC}(p, q)$ and $\mathrm{AC}$ components (corresponding to the harmonic distortion $)(p, q)$. From this, the current reference signal is

$\left[\begin{array}{l}I_{a}^{*} \\ I_{b}^{*} \\ I_{c}^{*}\end{array}\right]=\sqrt{\frac{2}{3}}\left[\begin{array}{cc}\frac{1}{-1} & 0 \\ \frac{-1}{2} & \frac{\sqrt{3}}{2} \\ \frac{-1}{2} & -\frac{\sqrt{3}}{2}\end{array}\right]\left[\begin{array}{cc}V_{\alpha} & V_{\beta} \\ V_{\beta} & -V_{\beta}\end{array}\right]^{-1}\left[\begin{array}{l}\tilde{p} \\ \tilde{q}\end{array}\right]$

The Fig. 4 shows a basic model in order to implement the PQ algorithm in Shunt Active Power Filter of proposed model. The p-q Block and the Inverse p-q Block bears the subsequent algorithms to the equation (2-5). As PQ algorithm is a power based algorithms the load current also has its role at the input side. Ploss input shown in the figure is correction which is due to the losses incurred in the switching of the VSI and it has to be taken into consideration.

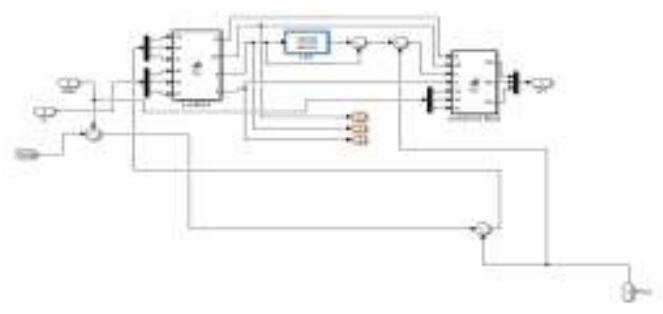

Fig. 4 MATLAB/SIMULINK model using PQ algorithm

\section{E. CSA with RLS Algorithm}

Conventional search methods have long been applied to solve engineering design problems. Although these methods find promising results in many real problems, they may fail in more complex design problems.

Crows (crow family or corvids) are considered the most intelligent birds. They contain the largest brain relative to their body size. Based on a brain-to-body ratio, their brain is slightly lower than a human brain. Evidences of the cleverness of crows are plentiful. They have demonstrated self-awareness in mirror tests and have tool-making ability. Crows can remember faces and warn each other when an unfriendly one approaches. Moreover, they can use tools, communicate in sophisticated ways and recall their food's hiding place up to several months later.

In this method the optimization of the errors calculated at the nonlinear loading point is done and further the correction is being achieved by the RLS algorithm. The THD calculation is the objective function and the entire crow flocks towards reducing the value keeping the magnitude of the signal intact. 
The system undergoes two major steps that is optimization and correction that is being achieved by the CSA and RLS working algorithms respectively.

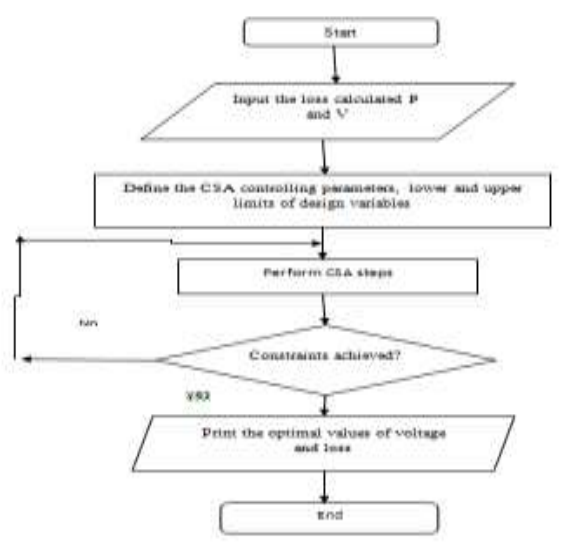

Fig. 5 Designing of proposed optimization at first phase

In real design problems, the number of decision variables can be very large and their effect on the objective function can be very complicated. The objective function may have many local optima, whereas the designer is interested in the global optimum. Such problems cannot be handled by conventional methods that only find local optima. In these cases, efficient optimization methods are needed.

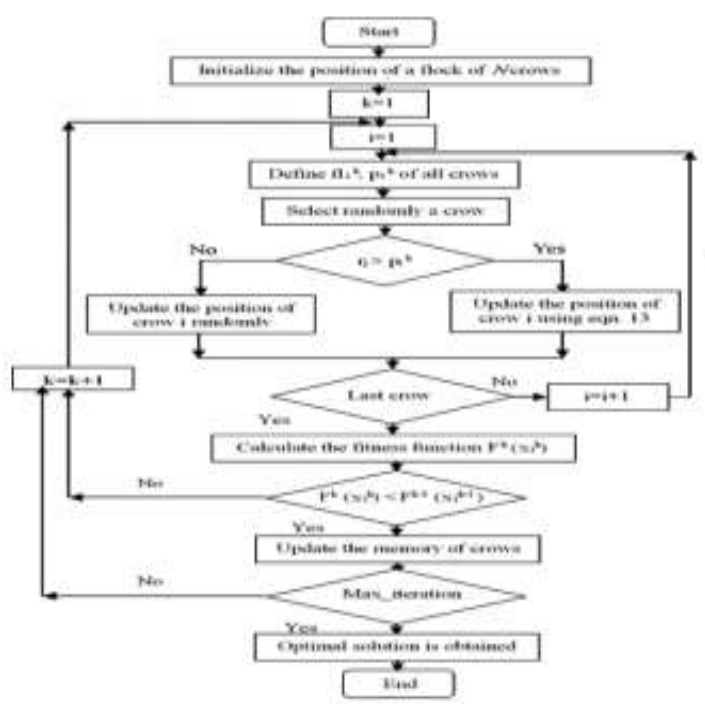

Fig. 6 Algorithm written in MATLAB Script

Recursive least squares is an adaptive filter algorithm that recursively finds the coefficients that minimize a weighted linear least squares cost function relating to the input signals.

Generation of Compensating Current using RLS Algorithm is used in HAPF so as to get DC link active current parameter, maintaining DC link voltage with the constant value of each phase and to make ripple free in the waveform.

The value of the instantaneous active current can be obtained from the HAPF in order to compensate for the loss of power and the difference in active power between the load and the supply network. The operation of the RLS algorithm, in which the voltage of the reference intermediate circuit and the voltage of the intermediate circuit Vcc (i) have a current sample.

In source voltage, harmonics are not present, so instantaneous voltage given as

$V_{x}(t)=V_{a x}(t)$

For each phase, the reference active current is given as

$i_{a x}(t)=A_{x} V_{x}(t)$

The instantaneous reference compensating current specified as $i(t)=i_{x}(t)-i_{a x}(t)$

RLS algorithms are known for their exceptional performance in variable time environments, but at the cost of increased processing complexity and stability problems. In this algorithm, the filter tap weighting vector is calculated using the Equations below

$$
\begin{aligned}
& w(n)=\bar{w}^{T}(n-1)+k(n) \overline{e_{n-1}}(n) \\
& k(n)=u(n) /\left(\lambda+X^{1}(n) u(n)\right) \\
& u(n)=\overline{w_{\lambda}^{-1}}(n-1) X(n)
\end{aligned}
$$

Eq. (9) and (10) are intermediate gain vector used to compute tap weights. Where $\lambda$ is a small positive constant very close to, but smaller than 1 .

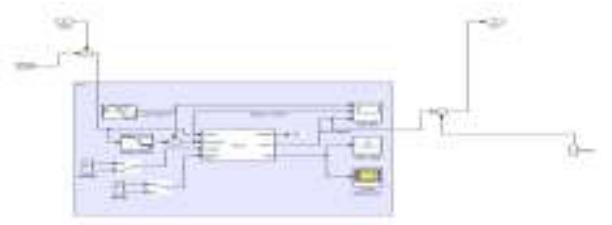

Fig. 7 RLS corrective measure after the optimization by the CSA.

\section{F. Hybrid Algorithm}

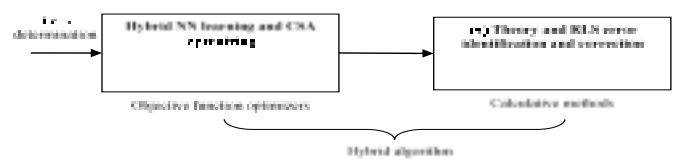

Fig. 8 Architecture of hybrid algorithm proposed 
The oulses provided to the power filter for operation has been geenrated by making use of the hybrid algorithms. The first part is the optimisation of the errors calculated at the voltgae and currrent points where two methods namely $\mathrm{NN}$ learning and the CSA procedure is being adopted. The method of NN learning is the one where the system is trained with the require output parametres and changes at the loading points via minimum 100 iterations and then the CSA optimising step takes place. In CSA the THD\% reduction has been focussed and taken as bjective function to be minimised. The second part is the corrective calculation methods where the hybridisation of PQ theiry with RLS based method has been done. This can be considered as a modifiedform of the recursive least square algorithm. Here in this methodology voltage distortion is removed further more effciently by using two types of optimisung algorithm. The error signals are traced using RLS algorithm and PQ algorithm both one by one to make the harmonics reduction more proficient.

Neural network (NN) has been employed in many applications in recent years. An NN is an interconnection of a number of artificial neurons that simulates a biological brain system. NN have been successfully introduced into power electronics circuits to generate the optimal switching angles of a PWM for a given modulation index. Neural network deals with Mathematical information about processing of a system with input and output. The neurons are trained using the Neural Network toolbox of MATLAB.

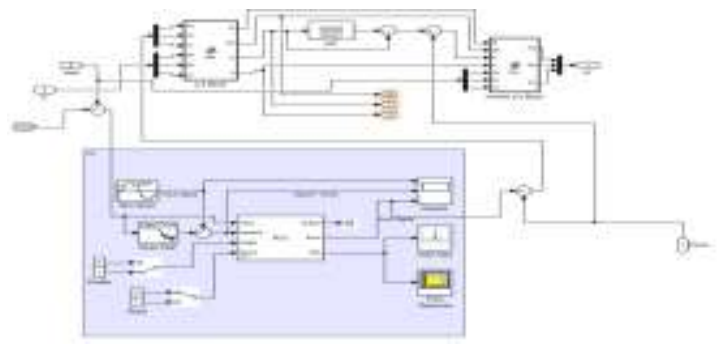

Fig. 9 MATLAB/SIMULINK model of hybrid PQ_RLS corrective Method

The figure above depicts MATLAB/SIMULINK model of control of power filter based on PQ_RLS algorithm. The difference in the voltage output from the source and voltage available at the load terminal is the error signal which is taken as input to the RLS algorithm. The optimum value of the output from RLS algorithm is further compared with any kind of losses in power converter. This output is then optimized via PQ algorithm and the output is being directed to generate pulses for the power filter operation.

\section{G. Pulse Generation}

The Pulse Generator block generates square wave pulses at regular intervals. The block's waveform parameters, Amplitude, Pulse Width, Period, and Phase Delay, determine the shape of the output waveform. The following figure shows how the pulses for triggering switches of VSI being generated. The Fig 3.7 shows $\mathrm{Vr}$ which is output of the compensating calculation (involves control algorithms) gets converted to the gating pulses using band controller.

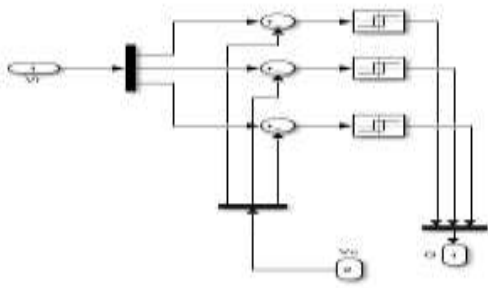

Fig. 10 MATLAB/SIMULINK Pulse generating model

V. Simulation Results and Distortion Calculation

A. The Result analysis of the model has been arranged in this study in the following manner:

1. CASE 1: Analysis of load line parameters

2. CASE 2: Analysis of voltage output from the power filter to be injected into line.

3. Validation

The table 1 below describes the system parameters of the source that has been feeding the load via power filters.

\begin{tabular}{|l|l|}
\hline \multicolumn{2}{|c|}{ Table 1 System Parameters: } \\
\hline \multicolumn{2}{|c|}{ Voltage Source Inverter } \\
\hline Phase to phase voltage (Volts) & 320 \\
\hline Frequency (Hertz) & 50 \\
\hline Source resistance (ohms) & 0.001 \\
\hline Source Inductance (H) & $1 \times 10^{-8}$ \\
\hline \multicolumn{2}{|c|}{ Power Filter } \\
\hline Capacitor Initial voltage (Volts) & 65 \\
\hline Capacitor initial capacitance (F) & $470 \times 10^{-8}$ \\
\hline
\end{tabular}

The model has been created in MATLAB having three phase voltage source as its main supply. The end consists of linear and nonlinear loads. The system under test has approximately 320 volts voltage in the transmission line. The power filer is designed for the same line using different algorithms. The three algorithms have been taken being PQ, RLS and PQ_RLS control.

The power filter in model shown in figure 1 comprises of all the elements as discussed. For the sake of comparison everything other than power filter is similar in all the three cases as discussed above. 


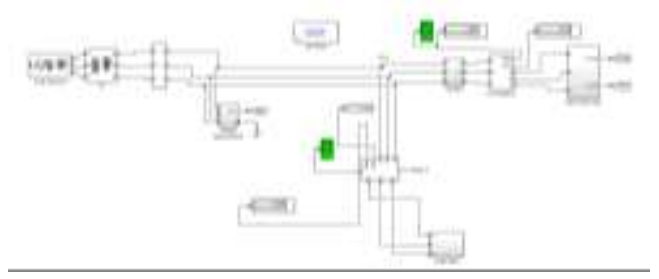

Fig. 11 The System under Test Comprising of Shunt Active Power Filter with AI algorithms testing.

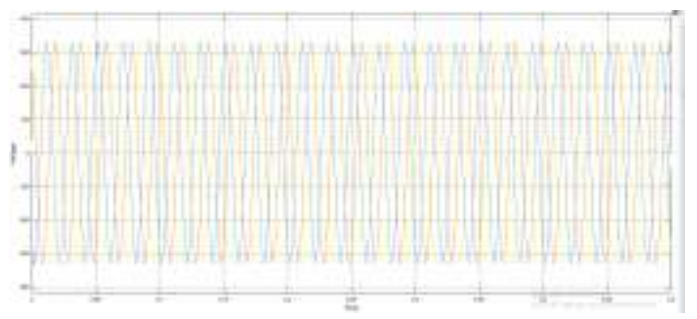

Figure 12 Three phase voltage in the transmission line by the source.

After proper analysis of the system with the power filter and its control algorithm the conclusion about the best optimizing technique has been made.

\section{B. CASE 1: Analysis of load line parameters}

Power filter designed with the online based-PQ theory for filtration at load line.

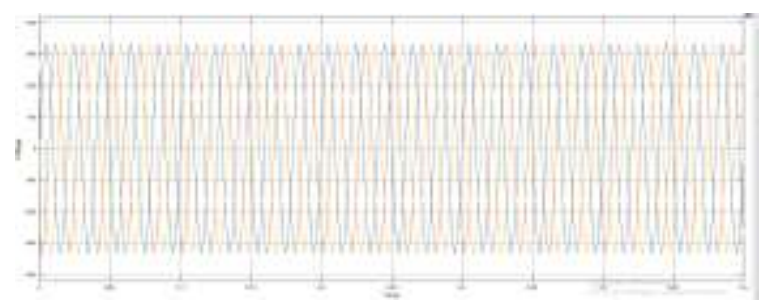

Fig. 13 Voltage at the load line in the system having filter with PQ theory control.

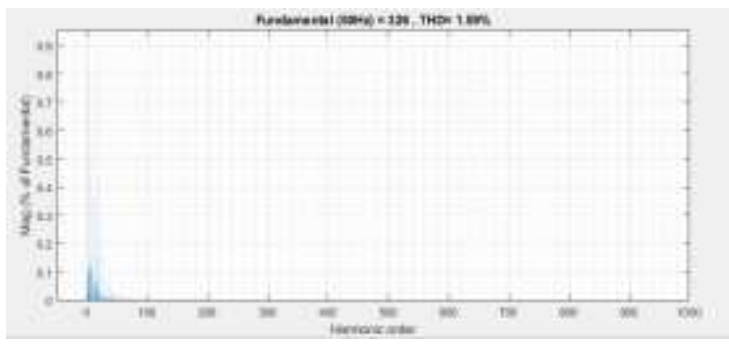

Fig. 14 THD\% of the load line voltage in the system with PQ theory control

The system under test is modeled with the power filter having PQ theory based online linear control algorithm and the analysis of voltage available at the nonlinear is studied. The above graphs depict the FFT analysis of the voltage available at the nonlinear load terminal and the THD in it is found to be $1.59 \%$.

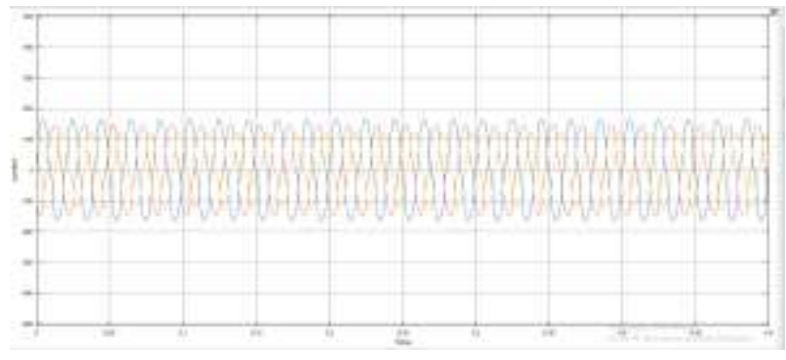

Fig. 15 Voltage at the load line in the system having filter with PQ theory control

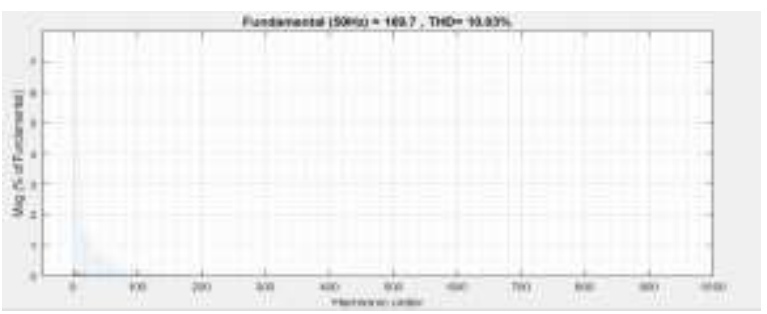

Fig. 16 THD\% in Voltage at the load line in the system having filter with PQ theory control

The system with power filter under test is also studied for the current waveform with the basic PQ theory based control algorithm and with the analysis it is concluded that the THD\% in current is found to be $10.03 \%$ at the terminal of nonlinear load. With a non-linear load the current isn't proportional to the voltage and it fluctuates based on the alternating load impedance.

Load analysis with Modified PQ theory with adaptive recursive least square (RLS) and crow optimizing algorithm for filter

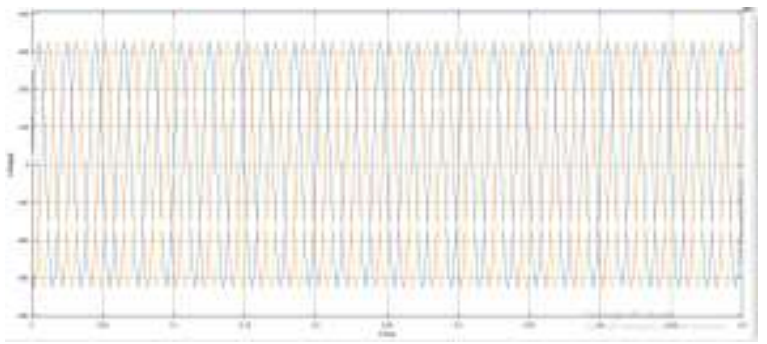

Fig. 17 Voltage available at the load line in system having adaptive recursive least square and crow optimizing algorithm

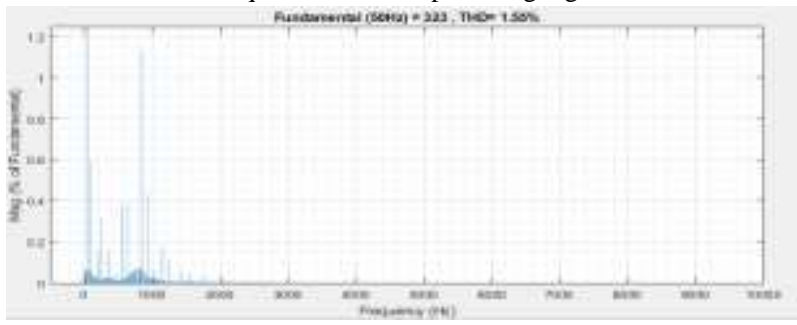

Figure 18 THD\% in voltage load line with adaptive recursive least square and crow optimizing algorithm 
The power filter in this case has a controller which is designed with the optimizing crow search algorithm with THD\% as its objective function. The work has been carried out with the adaptive recursive least square algorithm for further enhancement in the voltage waveform. The above graphs depict the FFT analysis of the voltage available at the nonlinear load terminal and therefore the THD in it is found to be $1.55 \%$.

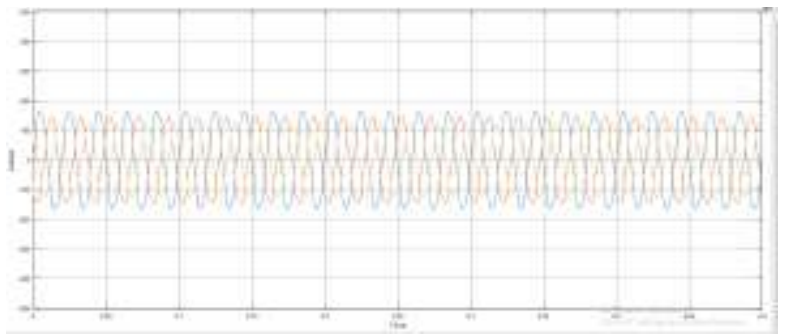

Fig. 19 current drawn at the load line in system having adaptive recursive least square and crow optimizing algorithm

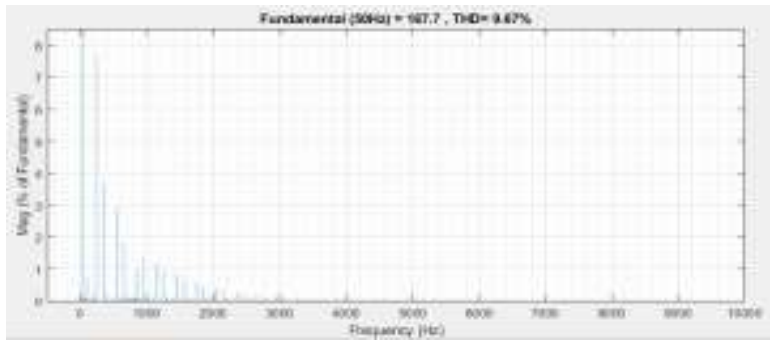

Fig. 20 THD\% in current drawn at the load line having CSA+ adaptive recursive least square technique

The system with power filter under test is also studied for the current waveform with the control algorithm having the AIbased crow optimizing technique and recursive least square calculation with the analysis it is concluded that the THD\% in current is found to be $9.67 \%$ at the terminal of nonlinear load. Load analysis PQ theory modified with recursive least square technique having hybrid crow search optimizer and $\mathrm{NN}$ learning technique for filter. The system having the power filter in this case has been modeled with a hybrid working algorithms for the THD\% enhancement. The modified algorithm depicted better results with previous NN learning technique.

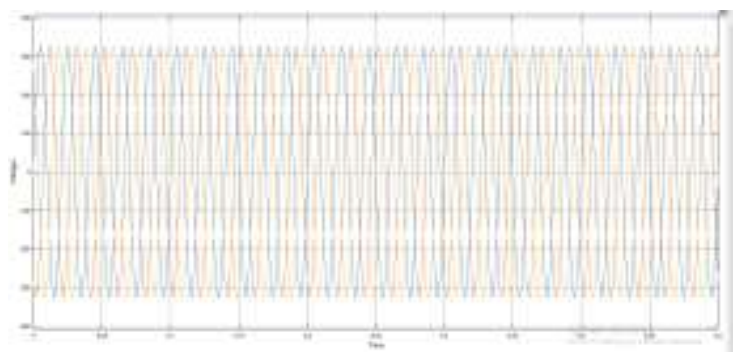

Fig. 21 Voltage at the load terminal with modified PQ theory with RLS algorithm and optimizing by crow search and $\mathrm{NN}$ learning

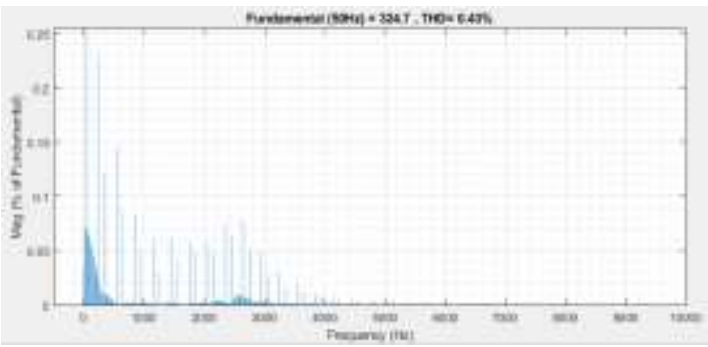

Fig. 22 THD\% in Voltage at the load terminal with modified PQ theory +RLS $+\mathrm{CSA}+\mathrm{NN}$

The system under tests depicts the result of hybrid algorithms meant for optimizing the voltage performance.

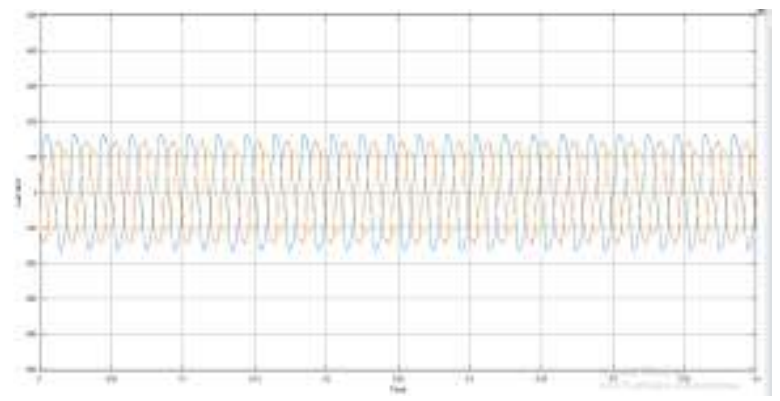

Fig. 23 Current at the load terminal with modified PQ theory with RLS algorithm and optimizing by crow search and nn learning

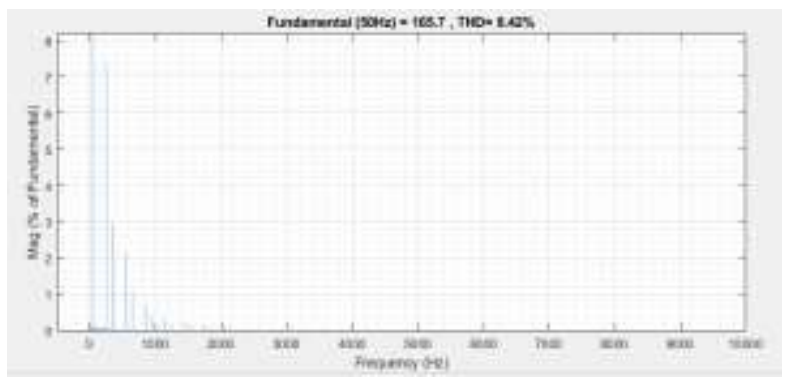

Fig. 24 THD \% of current at the load terminal with modified PQ theory +RLS $+\mathrm{CSA}+\mathrm{NN}$

The system with power filter under test is also studied for the current waveform with the control algorithm having the $\mathrm{NN}$ learning and thereby optimizing by the crow search algorithm. Further the losses are subjected to PQ theory and RLS corrective calculation. With the analysis it is concluded that the THD\% in current is found to be $8.42 \%$ at the terminal of nonlinear load.

\section{CASE 2: Analysis of voltage output from the power filter to be injected into line.}

Voltage from Power filter designed with the online based-PQ theory for filtration 


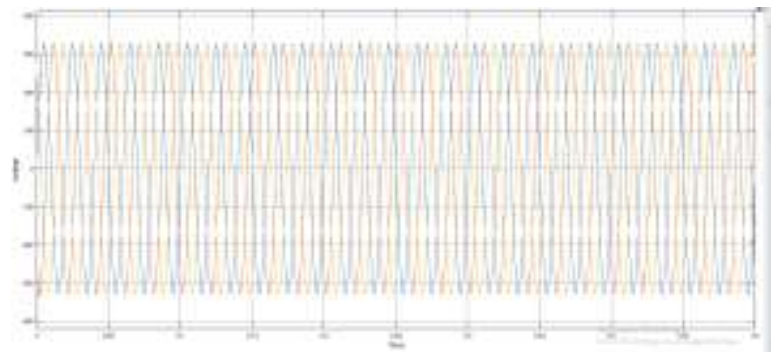

Figure 25 Voltage output from the hybrid power filter with PQ theory based control.

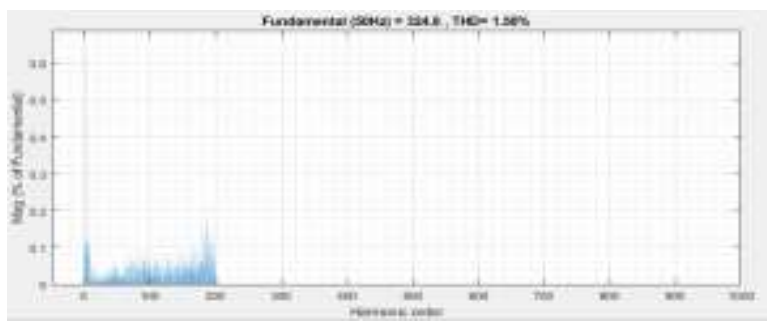

Fig. 26 THD\% in the Voltage output from the hybrid power filter with PQ theory based control

The system under test with power filter having PQ theory based controller for the power filter is studied for voltage output from the power filter .The graphs above shows FFT analysis of the voltage output from the power filter and THD\% level is calculated which was found to be $1.50 \%$

Voltage from adaptive recursive least square (RLS) and crow optimizing algorithm

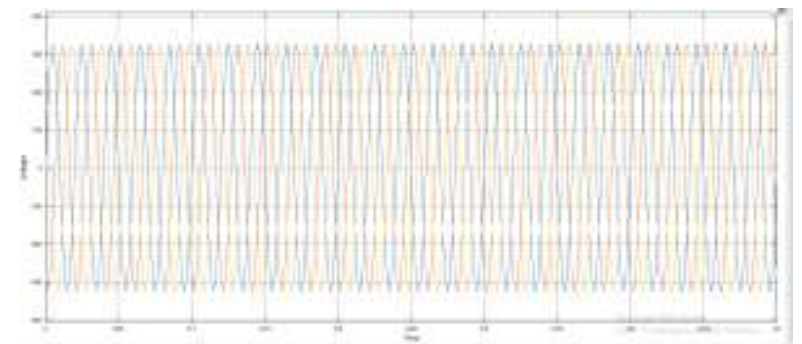

Figure 27 Voltage output from the power filter with RLS and CSA optimizing algorithm

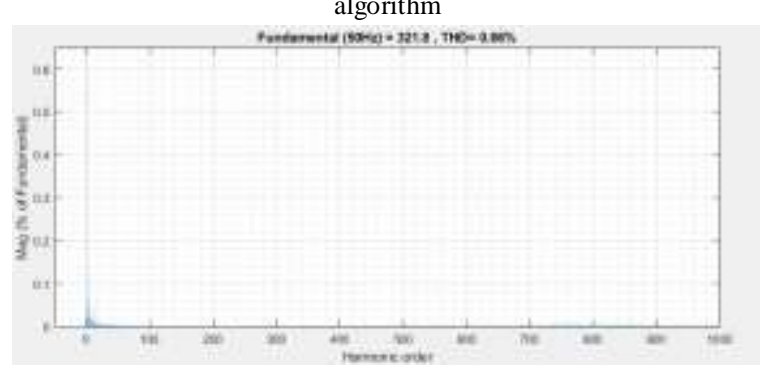

Fig. 28 THD\% in the Voltage output from the power filter with RLS and CSA optimizing algorithm

The system under test with power filter having modified RLS calculation after undergoing the loss optimizing by the crow search algorithm has been studied for the outcomes from the filter. The graphs above shows FFT analysis of the voltage output from the power filter and THD level is calculated which was found to be $0.66 \%$

Voltage from PQ theory modified with recursive least square technique having hybrid crow search optimizer and NN learning technique.

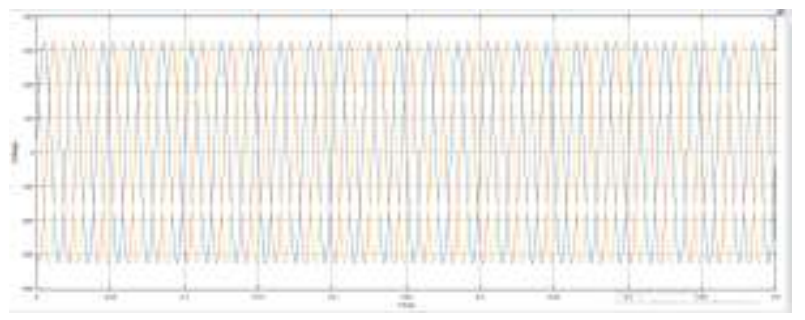

Fig. 29 Voltage output from the filter driven by hybrid controller

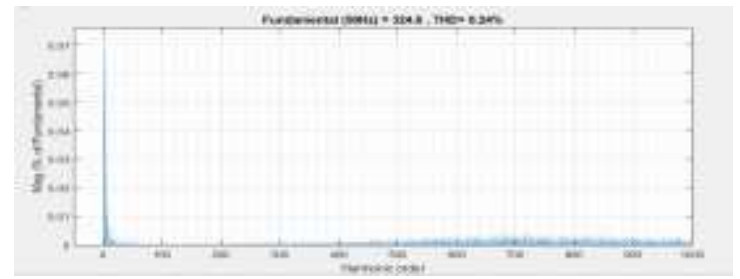

Fig. 30 THD\% in the Voltage output from the filter driven by hybrid controller

The system in this case has a hybrid optimizing technique as well as the hybrid calculative technique for more optimization in the process. The optimizing algorithm as been achieved by the crow search algorithm in combination with nn learning and further corrective calculation is done using the PQ theory with recursive least square method. The graphs above shows FFT analysis of the voltage output from the power filter and THD level is calculated which was found to be $0.24 \%$

\section{Validation}

The THD analysis of two waveforms has been done in this work. One at the voltage input to the nonlinear load and other of the voltage output from the power filter after using three types of algorithms.

This concludes that the proposed algorithm Having the hybrid optimizing and hybrid corrective algorithm in third case has been proved more effective in reducing the distortion level. The algorithm makes use of NN learning technique for deciding the corrective factors and then is further optimized with the crow search algorithm.

The reference generated further under goes corrective calculation and comparison using the hybrid PQ theory and Recursive least square learning (RLS) mechanism. 


\begin{tabular}{|l|c|c|}
\hline \multicolumn{2}{|c|}{$\begin{array}{c}\text { Table 2 The THD\% calculation at the various terminals } \\
\text { using various power filter driving algorithms }\end{array}$} \\
\hline $\begin{array}{c}\text { Controlling } \\
\text { techniques for the } \\
\text { filter }\end{array}$ & $\begin{array}{c}\text { THD\% of } \\
\text { voltage at the } \\
\text { load terminal }\end{array}$ & $\begin{array}{c}\text { THD\% in voltage } \\
\text { output from the } \\
\text { power filter }\end{array}$ \\
\hline $\begin{array}{l}\text { Power filter with } \\
\text { PQ theory based } \\
\text { controller }\end{array}$ & $1.59 \%$ & $1.50 \%$ \\
\hline $\begin{array}{l}\text { CSA and RLS } \\
\text { based filter }\end{array}$ & $1.55 \%$ & $0.66 \%$ \\
\hline $\begin{array}{l}\text { Hybrid algorithm } \\
\text { based filter having } \\
\text { NN+CSA } \\
\text { optimizing and } \\
\text { PQ+RLS } \\
\text { correction }\end{array}$ & $0.43 \%$ & $0.24 \%$ \\
\hline
\end{tabular}

The distortion level at the terminals were found to be $0.43 \%$ at the loading terminal with the hybrid controller and the output distortion level of the filter with this controller was having a distortion of $0.24 \%$.

\begin{tabular}{|l|l|}
\hline \multicolumn{2}{|c|}{ Table 3 THD in current at the load terminal } \\
\hline Power filter with PQ theory based controller & $10.03 \%$ \\
\hline CSA and RLS based filter & $9.67 \%$ \\
\hline $\begin{array}{l}\text { Hybrid algorithm based filter having } \\
\text { NN+CSA optimizing and PQ+RLS } \\
\text { correction }\end{array}$ & $8.42 \%$ \\
\hline
\end{tabular}

The THD analysis of current waveform is also done. This concludes that the algorithm in the third case having the hybrid controller has been proved more effective in reducing the distortion level in case of current at the load terminal also.

The least distortion in the current waveform was in the case of the hybrid controlling technique chosen for the filter having the AI based optimizing algorithms. The distortion was found to be $8.42 \%$ in the current drawn at the loading terminal of the nonlinear load.

\section{CONCLUSION}

In this work a power filter has been designed using different algorithms with an objective to reduce the Total Harmonic Distortion in the voltage output waveforms. The following major points we concluded from the above analysis:
- The total harmonic distortion in the voltage output waveform being fed to the load using only PQ theory based online learning algorithm is found to be $1.59 \%$.

- In case of the output voltage at the load terminal from the power buffer using proposed hybrid algorithm comprising of NN learning with CSA optimizing control and PQ_RLS algorithm for THD reduction the THD\% level is $0.43 \%$.

- The distortion level in the output voltage waveforms in both the cases being fed to the load when compared, it is found that proposed hybrid structure of control for the filter is more effective in reducing the distortion as compared to standard PQ theory based architecture.

- The distortion level at the current has been considerably reduced in order to get waveform that would be able to drive the load, both linear and nonlinear more effectively and efficiently.

\section{VII.SUGGESTION FOR FUTURE WORK}

Energy quality can be improved by using a more efficient power filter and algorithm in a networked system. The work can be expanded to improve the quality of the network powered by renewable energy sources. By improving the signal quality of the output voltage, it can be further integrated into the network.

The modulation technique is simple and easy to implement. Using real facts can make the UPS more robust and easier. With the advent of more powerful DPS, the computational complexity and the memory usage needs of the algorithms will decrease and more sophisticated and efficient algorithms can be implemented. It is therefore certainly true that the field of adaptive filtering will remain a long-open field for scientific research and commercial applications.

\section{REFERENCES}

[1] V Taraka Rama Reddy, T Siva Sai "Harmonic Mitigation and Power Quality Enhancement Using PV Fed Series Active Filter for Grid Systems" international journal of scientific \& technology research volume 9, issue 01, january 2020.

[2] Abdul Kahar, N, Zobaa, A (2019) Application of mixed integer distributed ant colony optimization to the design of undamped singletuned passive filters based harmonics mitigation. Swarm and Evolutionary Computation 44:187-199.

[3] Arunsankar, G, Srinath, S (2019) Optimal controller for mitigation of harmonics in hybrid shunt active power filter connected distribution system: An EGOANN technique. Journal of Renewable and Sustainable Energy 11(2): 1-16.

[4] Buch, H, Trivedi, I (2019) An efficient adaptive moth flame optimization algorithm for solving large-scale optimal power flow problem with POZ, multifuel and valve-point loading effect. Iranian Journal of Science and Technology, Transactions of Electrical Engineering 43(4): 1031-1051.

[5] Jiale Zhou, Yong Yang "Control Strategies of Mitigating Dead-time Effect on Power Converters: An Overview” Electronics 2019, 8(2), 196. 
[6] A.Sivakumar, N.B.Muthu Selvan Reduction of source current harmonics in ANN controlled induction motor, Alexandria Engineering Journal, Volume 57, Issue 3, September 2018, Pages 1489-1499

[7] Biswas, P, Suganthan, P, Amaratunga, G (2017) Minimizing harmonic distortion in power system with optimal design of hybrid active power filter using differential evolution. Applied Soft Computing 61: 486-496.

[8] Naresh Malla, Dipesh Shrestha "Online Learning Control for Harmonics Reduction Based on Current Controlled Voltage Source Power Inverters" IEEE/CAA Journal of Automatica Sinica, Vol. 4, No. 3, July 2017

[9] Das, S, Ray, P, Mohanty, A (2017) Improvement in power quality using hybrid power filters based on RLS algorithm. Energy Procedia 138: 723-728.

[10] Rangarajan, S, Collins, E, Fox, J (2019) Efficacy of a smart photovoltaic inverter as a virtual detuner for mitigating network harmonic resonance in distribution systems. Electric Power Systems Research 171: 175-184. 